PROCEEDINGS OF THE

AMERICAN MATHEMATICAL SOCIETY

Volume 136, Number 7, July 2008, Pages 2547-2554

S 0002-9939(08)09311-8

Article electronically published on January 24, 2008

\title{
GLOBAL BEHAVIOR OF THE BRANCH OF POSITIVE SOLUTIONS TO A LOGISTIC EQUATION OF POPULATION DYNAMICS
}

\author{
TETSUTARO SHIBATA \\ (Communicated by Carmen C. Chicone)
}

\begin{abstract}
We consider the nonlinear problem arising in population dynamics:

$-u^{\prime \prime}(t)+u(t)^{p}=\lambda u(t), u(t)>0, \quad t \in I:=(0,1), u(0)=u(1)=0$,

where $p>1$ is a constant and $\lambda>0$ is a positive parameter. We establish the crucial asymptotic formula for the branch of positive solutions $\lambda_{q}(\alpha)$ in $L^{q}$-framework as $\alpha \rightarrow \infty$, where $\alpha:=\left\|u_{\lambda}\right\|_{q}(1 \leq q<\infty)$. Especially, for the original logistic equation, namely the case where $p=2$ and $q=1$, we obtain not only the asymptotic expansion formula for $\lambda_{1}(\alpha)$ but also the remainder estimate. Such a formula for the bifurcation branch seems to be new.
\end{abstract}

\section{INTRODUCTION}

We consider the following nonlinear problem arising in population dynamics:

$$
\begin{aligned}
-u^{\prime \prime}(t)+u(t)^{p} & =\lambda u(t), \quad t \in I:=(0,1), \\
u(t) & >0, \quad t \in I, \\
u(0) & =u(1)=0
\end{aligned}
$$

where $p>1$ is a constant and $\lambda>0$ is a positive parameter. We know from 1 that for each given $\alpha>0$, there exists a unique solution $(\lambda, u)=\left(\lambda_{q}(\alpha), u_{\alpha}\right) \in$ $\mathbf{R}_{+} \times C^{2}(\bar{I})$ of (1.1)-(1.3) with $\left\|u_{\alpha}\right\|_{q}=\alpha$, where $\|\cdot\|_{q}$ denotes the $L^{q}$-norm. The set $\left\{\left(\lambda_{q}(\alpha), u_{\alpha}\right) ; \alpha>0\right\}$ gives all solutions of (1.1)-(1.3) and is an unbounded curve of class $C^{1}$ in $\mathbf{R}_{+} \times C^{2}(\bar{I})$ emanating from $\left(\pi^{2}, 0\right)$. The curve $\lambda_{q}(\alpha)(\alpha>0)$ is called the bifurcation branch of positive solutions in $L^{q}$-framework.

The purpose of this paper is to establish the asymptotic expansion formula for $\lambda_{q}(\alpha)$ as $\alpha \rightarrow \infty$ to understand the global behavior of the bifurcation branch well.

(1.1)-(1.3) has been investigated by many authors from the viewpoint of $L^{\infty}$ framework and $L^{2}$-framework, since (1.1)-(1.3) is regarded as a bifurcation problem and a nonlinear eigenvalue problem. We refer to [1], 2], 3], 4], 5, 6], 7] and 8 for the works in these directions. A further important viewpoint is that (1.1)-(1.3) is a model equation of population density for some species when $p=2$. Here, $\lambda>0$ is considered as the reciprocal number of its diffusion rate.

Received by the editors June 8, 2007.

2000 Mathematics Subject Classification. Primary 34B15.

Key words and phrases. $L^{q}$-bifurcation branch, asymptotic formula.

(C)2008 American Mathematical Society Reverts to public domain 28 years from publication 
From the standpoint of this biological background, one of the most crucial problems to consider is the asymptotic behavior of $\lambda_{1}(\alpha)$, which describes the relationship between the total number of population and the reciprocal number of its diffusion rate.

It is well known (cf. [1]) that for $t \in I$, as $\alpha \rightarrow \infty$,

$$
\frac{u_{\alpha}(t)}{\lambda_{q}(\alpha)^{1 /(p-1)}} \rightarrow 1
$$

This implies that as $\alpha \rightarrow \infty$,

$$
\lambda_{q}(\alpha)=\alpha^{p-1}(1+o(1)) .
$$

Recently, the $L^{q}$-bifurcation diagram of (1.1)-(1.3) with $u^{p}$ replaced by a more general nonlinear term $f(u)$ was considered in [9]. As a corollary of the result obtained there, (1.5) was improved as follows.

Theorem 1.1 ([9]). Consider (1.1)-(1.3). Let $1 \leq q<\infty$ be fixed. Then as $\alpha \rightarrow \infty$

$$
\lambda_{q}(\alpha)=\alpha^{p-1}+C_{1} \alpha^{(p-1) / 2}+o\left(\alpha^{(p-1) / 2}\right),
$$

where

$$
\begin{aligned}
& C_{1}=\frac{p-1}{q} C(q), \\
& C(q):=2 \int_{0}^{1} \frac{1-s^{q}}{\sqrt{1-s^{2}-2\left(1-s^{p+1}\right) /(p+1)}} d s .
\end{aligned}
$$

Since general nonlinear term $f(u)$, which included $u^{p}$, was considered in [9], it was difficult to obtain the third term in (1.6) in 9 .

Here we focus on the nonlinear term $f(u)=u^{p}$ and obtain the almost complete asymptotic formula for $\lambda_{q}(\alpha)$ as $\alpha \rightarrow \infty$.

Now we state our results.

Theorem 1.2. Let $1 \leq q<\infty$ be fixed. Further, let an arbitrary positive integer $N$ be fixed. Then as $\alpha \rightarrow \infty$

$$
\lambda_{q}(\alpha)=\alpha^{p-1}+C_{1} \alpha^{(p-1) / 2}+a_{0}+\sum_{k=1}^{N} a_{k} \alpha^{k(1-p) / 2}+o\left(\alpha^{N(1-p) / 2}\right),
$$

where

$$
a_{0}=\frac{p-1}{2 q} C(q)^{2}, \quad a_{1}=\frac{(p-1)(p-1-2 q)(p-1-4 q)}{24 q^{3}} C(q)^{3}
$$

and $\left\{a_{j}\right\}_{j=1}^{N}$ is a constant determined by $C(q), a_{0}, a_{1}, \cdots, a_{j-1}$ inductively.

Especially, for the case $p=q+1$, which includes the original logistic case $p=2$ and $q=1$, we have the optimal remainder estimate.

Theorem 1.3. Let $p=q+1$. Then as $\alpha \rightarrow \infty$

$$
\begin{array}{ll}
\lambda_{1}(\alpha)= & \alpha^{p-1}+C(q) \alpha^{(p-1) / 2}+\frac{1}{2} C(q)^{2}+\sum_{n=1}^{\infty}\left(\begin{array}{c}
1 / 2 \\
n
\end{array}\right) \frac{C(q)^{2 n+1}}{2^{2 n}} \alpha^{-(p-1)(n-1 / 2)} \\
& +O\left(\alpha^{p-1} e^{-\sqrt{(p-1) \alpha^{p-1}(1+o(1))} / 2}\right) .
\end{array}
$$


Remark 1.4. When $q=2,(1.7)$ has been obtained in 8 . The main tool used there is the relationship between $\lambda_{2}(\alpha)$ and the critical value associated with $u_{\alpha}$, which only holds for the case $q=2$.

We prove Theorems 1.2 and 1.3 by quite an elementary and straightforward way, and do not use any complicated tools like those used in [8]. Here we compare $\left\|u_{\lambda}\right\|_{q}$ with $\left\|u_{\lambda}\right\|_{\infty}$ by direct calculation.

\section{Proof of the theorems}

Let $1 \leq q<\infty$ be fixed. In this section, we use the following notation. Let $\left(\lambda, u_{\lambda}\right) \in \mathbf{R}_{+} \times C^{2}(\bar{I})$ be the solution of (1.1)-(1.3) for given $\lambda>\pi^{2}$. Therefore, $\alpha=\left\|u_{\lambda}\right\|_{q}$. We write $\lambda=\lambda_{q}(\alpha)$ for simplicity. It is well known that for $\lambda \gg 1$,

$$
\lambda=\left\|u_{\lambda}\right\|_{\infty}^{p-1}+\lambda e^{-\sqrt{(p-1) \lambda}(1+o(1)) / 2} .
$$

For the reader's convenience, a simple proof of (2.1) will be given in the Appendix.

Proposition 2.1 below plays an important role in the proof of the theorems.

Proposition 2.1. As $\lambda \rightarrow \infty$

$$
\left\|u_{\lambda}\right\|_{q}^{p-1}=\lambda\left(1-\frac{C(q)}{\sqrt{\lambda}}\right)^{(p-1) / q}+O\left(\lambda e^{-\sqrt{(p-1) \lambda}(1+o(1)) / 2}\right) .
$$

We accept Proposition 2.1 tentatively and prove the theorems. The proof of Proposition 2.1 will be given in Section 3 .

Proof of Theorem 1.2. We have only to show how to obtain $a_{0}$ and $a_{1}$ in (1.7) by using (1.6) and Proposition 2.1. The coefficient $a_{k}(k=2, \cdots, N)$ can be obtained inductively by using the same argument as that to obtain $a_{0}$ and $a_{1}$.

(i) We calculate $a_{0}$. Let

$$
r(\alpha):=\lambda-\alpha^{p-1}-\frac{p-1}{q} C(q) \alpha^{(p-1) / 2} .
$$

By (1.6), $r(\alpha)=o\left(\alpha^{(p-1) / 2}\right)$ for $\alpha \gg 1$. By (2.2) and the Taylor expansion, for $\lambda \gg 1$

$$
\sqrt{\lambda}=\alpha^{(p-1) / 2}+\frac{p-1}{2 q} C(q)+o(1)
$$

By this, (2.2), (2.3) and the Taylor expansion,

$$
\begin{aligned}
\alpha^{p-1}= & \lambda\left(1-\frac{p-1}{q} \frac{C(q)}{\sqrt{\lambda}}+\frac{(p-1)(p-1-q)}{2 q^{2}} \frac{C(q)^{2}}{\lambda}+o\left(\lambda^{-1}\right)\right) \\
& +O\left(\lambda e^{-\sqrt{(p-1) \lambda}(1+o(1)) / 2}\right) \\
= & \lambda-\frac{p-1}{q} C(q) \sqrt{\lambda}+\frac{(p-1)(p-1-q)}{2 q^{2}} C(q)^{2}+o(1) \\
= & \alpha^{p-1}+\frac{p-1}{q} C(q) \alpha^{(p-1) / 2}+r(\alpha) \\
& -\frac{p-1}{q} C(q)\left(\alpha^{(p-1) / 2}+\frac{p-1}{2 q} C(q)+o(1)\right) \\
& +\frac{(p-1)(p-1-q)}{2 q^{2}} C(q)^{2}+o(1) .
\end{aligned}
$$


By this, for $\alpha \gg 1$,

$$
r(\alpha)=\frac{p-1}{2 q} C(q)^{2}+o(1) .
$$

This implies $a_{0}=(p-1) C(q)^{2} /(2 q)$.

(ii) We calculate $a_{1}$. Let

$$
V(\alpha):=\lambda-\alpha^{p-1}-\frac{p-1}{q} C(q) \alpha^{(p-1) / 2}-\frac{p-1}{2 q} C(q)^{2} .
$$

By step (i), $V(\alpha)=o(1)$ for $\alpha \gg 1$. By (2.4) and the Taylor expansion, for $\lambda \gg 1$

$$
\begin{aligned}
\sqrt{\lambda}= & \alpha^{(p-1) / 2}+\frac{p-1}{2 q} C(q)+\frac{p-1}{4 q} C(q)^{2} \alpha^{-(p-1) / 2} \\
& -\frac{(p-1)^{2}}{8 q^{2}} C(q)^{2} \alpha^{-(p-1) / 2}+o\left(\alpha^{-(p-1) / 2}\right) .
\end{aligned}
$$

By this, (2.2), (2.4) and the Taylor expansion,

$$
\begin{aligned}
\alpha^{p-1}= & \lambda\left(1-\frac{p-1}{q} \frac{C(q)}{\sqrt{\lambda}}+\frac{(p-1)(p-1-q)}{2 q^{2}} \frac{C(q)^{2}}{\lambda}\right. \\
& \left.-\frac{(p-1)(p-1-q)(p-1-2 q)}{6 q^{3}} \frac{C(q)^{3}}{\lambda^{3 / 2}}(1+o(1))\right) \\
& +O\left(\lambda e^{-\sqrt{(p-1) \lambda}(1+o(1)) / 2}\right) \\
= & \lambda-\frac{p-1}{q} C(q) \sqrt{\lambda}+\frac{(p-1)(p-1-q)}{2 q^{2}} C(q)^{2} \\
& -\frac{(p-1)(p-1-q)(p-1-2 q)}{6 q^{3}} \frac{C(q)^{3}}{\lambda^{1 / 2}}(1+o(1)) \\
= & \alpha^{p-1}+\frac{p-1}{q} C(q) \alpha^{(p-1) / 2}+\frac{p-1}{2 q} C(q)^{2}+V(\alpha) \\
& -\frac{p-1}{q} C(q)\left(\alpha^{(p-1) / 2}+\frac{p-1}{2 q} C(q)+\frac{p-1}{4 q} C(q)^{2} \alpha^{-(p-1) / 2}\right. \\
& +\frac{(p-1)(p-1-q)}{2 q^{2}} C(q)^{2} \\
& \left.-\frac{(p-1)(p-1-q)(p-1-2 q)}{6 q^{2}} C(q)^{2} \alpha^{-(p-1) / 2}+o\left(\alpha^{-(p-1) / 2}\right)\right)
\end{aligned}
$$

By this, for $\alpha \gg 1$,

$$
V(\alpha)=\frac{(p-1)(p-1-2 q)(p-1-4 q)}{24 q^{3}} C(q)^{3} \alpha^{-(p-1) / 2}+o\left(\alpha^{-(p-1) / 2}\right) .
$$

This implies

$$
a_{1}=\frac{(p-1)(p-1-2 q)(p-1-4 q)}{24 q^{3}} C(q)^{3} .
$$

Thus the proof is complete.

Proof of Theorem 1.3. Since $p=q+1$, by $(2.2)$, for $\lambda \gg 1$

$$
\alpha^{p-1}=\lambda-C(q) \sqrt{\lambda}+O\left(\lambda e^{-\sqrt{(p-1) \lambda}(1+o(1)) / 2}\right) .
$$


This implies that

$$
\sqrt{\lambda}=\frac{\left.C(q)+\sqrt{C(q)^{2}-4\left(-\alpha^{p-1}+O\left(\lambda e^{-\sqrt{(p-1) \lambda}(1+o(1)) / 2}\right)\right.}\right)}{2} .
$$

By this,

$$
\begin{aligned}
\lambda= & \alpha^{p-1}+C(q) \sqrt{\alpha^{p-1}+\frac{C(q)^{2}}{4}}+\frac{1}{2} C(q)^{2}+O\left(\alpha^{p-1} e^{-\sqrt{(p-1) \alpha^{p-1}(1+o(1))} / 2}\right) \\
= & \alpha^{p-1}+C(q) \alpha^{(p-1) / 2}+\frac{1}{2} C(q)^{2}+\sum_{n=1}^{\infty}\left(\begin{array}{c}
1 / 2 \\
n
\end{array}\right) \frac{C(q)^{2 n+1}}{2^{2 n}} \alpha^{-(p-1)(n-1 / 2)} \\
& +O\left(\alpha^{p-1} e^{-\sqrt{(p-1) \alpha^{p-1}(1+o(1))} / 2}\right) .
\end{aligned}
$$

Thus the proof is complete.

\section{Proof of Proposition 2.1}

We begin with notation and the fundamental properties of $u_{\lambda}$. In what follows, $C$ denotes various positive constants independent of $\lambda \gg 1$ for simplicity. We know from [1] that for $\lambda>\pi^{2}$

$$
\begin{aligned}
& u_{\lambda}(t)=u_{\lambda}(1-t), \quad 0 \leq t \leq 1, \\
& u_{\lambda}\left(\frac{1}{2}\right)=\max _{0 \leq t \leq 1} u_{\lambda}(t)=\left\|u_{\lambda}\right\|_{\infty}, \\
& u_{\lambda}^{\prime}(t)>0, \quad 0 \leq t<\frac{1}{2} .
\end{aligned}
$$

For $\lambda>\pi^{2}$ and $0 \leq s \leq 1$, let

$$
\begin{aligned}
R_{\lambda}(s) & :=1-s^{2}-\frac{2}{p+1} \frac{\left\|u_{\lambda}\right\|_{\infty}^{p-1}}{\lambda}\left(1-s^{p+1}\right), \\
S_{\lambda}(s) & :=1-s^{2}-\frac{2}{p+1}\left(1-s^{p+1}\right), \\
U_{\lambda} & :=2 \int_{0}^{1} \frac{\left(1-s^{q}\right)\left(S_{\lambda}(s)-R_{\lambda}(s)\right)}{\sqrt{R_{\lambda}(s)} \sqrt{S_{\lambda}(s)}\left(\sqrt{R_{\lambda}(s)}+\sqrt{S_{\lambda}(s)}\right)} d s .
\end{aligned}
$$

Lemma 3.1. For $\lambda>\pi^{2}$

$$
\left\|u_{\lambda}\right\|_{\infty}^{q}-\left\|u_{\lambda}\right\|_{q}^{q}=\frac{1}{\sqrt{\lambda}}\left\|u_{\lambda}\right\|_{\infty}^{q}\left(C(q)+U_{\lambda}\right) .
$$

Proof. It follows from (1.1) that for $0 \leq t \leq 1$

$$
\frac{d}{d t}\left[\frac{1}{2} u_{\lambda}^{\prime}(t)^{2}-\frac{1}{p+1} u_{\lambda}(t)^{p+1}+\frac{1}{2} \lambda u_{\lambda}(t)^{2}\right]=0 .
$$

By this and (3.2), for $0 \leq t \leq 1$, we obtain

$$
\begin{aligned}
& \frac{1}{2} u_{\lambda}^{\prime}(t)^{2}-\frac{1}{p+1} u_{\lambda}(t)^{p+1}+\frac{1}{2} \lambda u_{\lambda}(t)^{2}=\mathrm{constant} \\
& =-\frac{1}{p+1}\left\|u_{\lambda}\right\|_{\infty}^{p+1}+\frac{1}{2} \lambda\left\|u_{\lambda}\right\|_{\infty}^{2} .
\end{aligned}
$$


We put

$$
\begin{aligned}
M_{\lambda}(\theta) & :=\lambda\left(\left\|u_{\lambda}\right\|_{\infty}^{2}-\theta^{2}\right)-\frac{2}{p+1}\left(\left\|u_{\lambda}\right\|_{\infty}^{p+1}-\theta^{p+1}\right), \\
Q_{\lambda}(s) & :=\lambda\left\|u_{\lambda}\right\|_{\infty}^{2}\left(1-s^{2}\right)-\frac{2}{p+1}\left\|u_{\lambda}\right\|_{\infty}^{p+1}\left(1-s^{p+1}\right) .
\end{aligned}
$$

By (3.3), (3.8) and (3.9), for $0 \leq t \leq 1 / 2$,

$$
u_{\lambda}^{\prime}(t)=\sqrt{M_{\lambda}\left(u_{\lambda}(t)\right)} .
$$

By (3.1), (3.2), (3.11) and putting $\theta=u_{\lambda}(t)$ and $s=\theta /\left\|u_{\lambda}\right\|_{\infty}$, we obtain

$$
\begin{aligned}
\left\|u_{\lambda}\right\|_{\infty}^{q}-\left\|u_{\lambda}\right\|_{q}^{q} & =2 \int_{0}^{1 / 2}\left(\left\|u_{\lambda}\right\|_{\infty}^{q}-u_{\lambda}^{q}(t)\right) \frac{u_{\lambda}^{\prime}(t)}{\sqrt{M_{\lambda}\left(u_{\lambda}(t)\right)}} d t \\
& =2 \int_{0}^{\left\|u_{\lambda}\right\|_{\infty}}\left(\left\|u_{\lambda}\right\|_{\infty}^{q}-\theta^{q}\right) \frac{1}{\sqrt{M_{\lambda}(\theta)}} d \theta \\
& =2 \frac{\left\|u_{\lambda}\right\|_{\infty}^{q}}{\sqrt{\lambda}} \int_{0}^{1} \frac{1-s^{q}}{\sqrt{Q_{\lambda}(s) /\left(\lambda\left\|u_{\lambda}\right\|_{\infty}^{2}\right)}} d s \\
& =2 \frac{\left\|u_{\lambda}\right\|_{\infty}^{q}}{\sqrt{\lambda}} \int_{0}^{1} \frac{1-s^{q}}{\sqrt{R_{\lambda}(s)}} d s \\
& =\frac{\left\|u_{\lambda}\right\|_{\infty}^{q}}{\sqrt{\lambda}}\left(2 \int_{0}^{1} \frac{1-s^{q}}{\sqrt{S_{\lambda}(s)}} d s+U_{\lambda}\right) \\
& =\frac{\left\|u_{\lambda}\right\|_{\infty}^{q}}{\sqrt{\lambda}}\left(C(q)+U_{\lambda}\right) .
\end{aligned}
$$

Thus the proof is complete.

\section{Lemma 3.2. For $\lambda \gg 1$}

$$
\left|U_{\lambda}\right| \leq C \sqrt{\lambda} e^{-\sqrt{(p-1) \lambda}(1+o(1)) / 2} .
$$

Proof. We put $\xi_{\lambda}:=\lambda-\left\|u_{\lambda}\right\|_{\infty}^{p-1}$. We know from [1] that $\xi_{\lambda}>0$ for $\lambda>\pi^{2}$. Let $0<\epsilon \ll 1$ be fixed. Then by the Taylor expansion, there exists a constant $0<\delta \ll 1$ such that for $\lambda \gg 1$ and $1-\epsilon \leq s \leq 1$

$$
\begin{aligned}
& S_{\lambda}(s) \geq(p-1-\delta)(1-s)^{2}, \\
& R_{\lambda}(s) \geq \frac{\xi_{\lambda}}{\lambda}(1-s)+(p-1-\delta)(1-s)^{2} .
\end{aligned}
$$

We put

$$
\begin{aligned}
U_{\lambda}= & U_{1, \lambda}+U_{2, \lambda} \\
:= & 2 \int_{0}^{1-\epsilon} \frac{\left(1-s^{q}\right)\left(S_{\lambda}(s)-R_{\lambda}(s)\right)}{\sqrt{R_{\lambda}(s)} \sqrt{S_{\lambda}(s)}\left(\sqrt{R_{\lambda}(s)}+\sqrt{S_{\lambda}(s)}\right)} d s \\
& \quad+2 \int_{1-\epsilon}^{1} \frac{\left(1-s^{q}\right)\left(S_{\lambda}(s)-R_{\lambda}(s)\right)}{\sqrt{R_{\lambda}(s)} \sqrt{S_{\lambda}(s)}\left(\sqrt{R_{\lambda}(s)}+\sqrt{S_{\lambda}(s)}\right)} d s .
\end{aligned}
$$


By this, (2.1), (3.4), (3.5), (3.14) and (3.15)

$$
\begin{aligned}
\left|U_{2, \lambda}\right| & \leq 2 \int_{1-\epsilon}^{1} \frac{\left(1-s^{q}\right)\left(1-s^{p+1}\right)\left(1-\|\left. u_{\lambda}\right|_{\infty} ^{p-1} / \lambda\right)}{R_{\lambda}(s) \sqrt{S_{\lambda}(s)}} d s \\
& \leq C \frac{\xi_{\lambda}}{\lambda} \int_{1-\epsilon}^{1} \frac{1}{\left(\xi_{\lambda} / \lambda\right)+(p-1-\delta)(1-s)} d s \\
& =C \frac{\xi_{\lambda}}{\lambda} \int_{0}^{\epsilon} \frac{1}{\left(\xi_{\lambda} / \lambda\right)+C v} d v \\
& \leq C \frac{\xi_{\lambda}}{\lambda}\left|\log \left(\frac{\xi_{\lambda}}{\lambda}\right)\right| \\
& \leq C \sqrt{\lambda} e^{-\sqrt{(p-1) \lambda}(1+o(1)) / 2} .
\end{aligned}
$$

Next, it is clear that $S_{\lambda}(s) \geq C, R_{\lambda}(s) \geq C$ for $0 \leq s \leq 1-\epsilon$ and $\lambda \gg 1$. By this, (2.1), (3.4) and (3.5)

$$
\left|U_{1, \lambda}\right| \leq C \int_{0}^{1-\epsilon}\left(1-s^{q}\right)\left(1-s^{p+1}\right)\left(1-\|\left. u_{\lambda}\right|_{\infty} ^{p-1} / \lambda\right) d s \leq C \frac{\xi_{\lambda}}{\lambda} .
$$

By this, (2.1), (3.16) and (3.17), we obtain (3.13). Thus the proof is complete.

Proof of Proposition 2.1. By (2.1), Lemmas 3.1 and 3.2, for $\lambda \gg 1$

$$
\begin{aligned}
\left\|u_{\lambda}\right\|_{q}^{p-1}= & \left\|u_{\lambda}\right\|_{\infty}^{p-1}\left(1-\frac{1}{\sqrt{\lambda}}\left(C(q)+U_{\lambda}\right)\right)^{(p-1) / q} \\
= & \left(\lambda-\xi_{\lambda}\right)\left(1-\frac{1}{\sqrt{\lambda}}\left(C(q)+U_{\lambda}\right)\right)^{(p-1) / q} \\
= & \lambda\left(1-\frac{\xi_{\lambda}}{\lambda}\right)\left[\left(1-\frac{1}{\sqrt{\lambda}} C(q)\right)^{(p-1) / q}+O\left(\frac{U_{\lambda}}{q \sqrt{\lambda}}\right)\right] \\
= & \lambda\left(1-e^{-\sqrt{(p-1) \lambda}(1+o(1)) / 2}\right) \\
& \quad \times\left[\left(1-\frac{1}{\sqrt{\lambda}} C(q)\right)^{(p-1) / q}+O\left(e^{-\sqrt{(p-1) \lambda}(1+o(1)) / 2}\right)\right]
\end{aligned}
$$

By this, we obtain Proposition 2.1. Thus the proof is complete.

\section{Appendix}

In this section, we prove (2.1) for the reader's convenience. To do this, we calculate $\xi_{\lambda}=\lambda-\left\|u_{\lambda}\right\|_{\infty}^{p-1}$. By (3.4)

$$
\begin{aligned}
& R_{\lambda}^{\prime}(s)=-2 s+2 \frac{\left\|u_{\lambda}\right\|_{\infty}^{p-1}}{\lambda} s^{p}, \\
& R_{\lambda}^{\prime \prime}(s)=-2+2 p \frac{\left\|u_{\lambda}\right\|_{\infty}^{p-1}}{\lambda} s^{p-1} .
\end{aligned}
$$

Let an arbitrary $0<\epsilon \ll 1$ be fixed. We know from [1] that $\xi_{\lambda}=O(1)$ for $\lambda \gg 1$. By this, (4.1), (4.2) and the Taylor expansion, for $\lambda \gg 1$ and $1-\epsilon \leq s \leq 1$,

$$
R_{\lambda}(s)=\left(2 \xi_{\lambda} / \lambda\right)(1-s)+(p-1)(1+o(1))(1-s)^{2} .
$$


By (3.11) and the same argument used to obtain (3.12),

$$
\begin{aligned}
\frac{1}{2} & =\int_{0}^{1 / 2} d t=\int_{0}^{1 / 2} \frac{u_{\lambda}^{\prime}(t)}{\sqrt{M_{\lambda}\left(u_{\lambda}(t)\right)}} d t \\
& =\int_{0}^{\left\|u_{\lambda}\right\|_{\infty}} \frac{1}{\sqrt{M_{\lambda}(\theta)}} d \theta=\frac{1}{\sqrt{\lambda}} \int_{0}^{1} \frac{1}{\sqrt{R_{\lambda}(s)}} d s \\
& =\frac{1}{\sqrt{\lambda}}\left\{\int_{0}^{1-\epsilon} \frac{1}{\sqrt{R_{\lambda}(s)}} d s+\int_{1-\epsilon}^{1} \frac{1}{\sqrt{R_{\lambda}(s)}} d s\right\} \\
& =\frac{1}{\sqrt{\lambda}}\left\{C_{\epsilon}+\int_{1-\epsilon}^{1} \frac{1}{\sqrt{R_{\lambda}(s)}} d s .\right\},
\end{aligned}
$$

where $C_{\epsilon}>0$ is a constant, which is bounded for $\lambda \gg 1$. We set $m:=(p-1)(1+$ $o(1))$. By (4.3),

$$
\begin{aligned}
& \int_{1-\epsilon}^{1} \frac{1}{\sqrt{R_{\lambda}(s)}} d s=\int_{1-\epsilon}^{1} \frac{1}{\sqrt{\left(2 \xi_{\lambda} / \lambda\right)(1-s)+m(1-s)^{2}}} d s \\
& =\int_{0}^{\epsilon} \frac{1}{\sqrt{\left(2 \xi_{\lambda} / \lambda\right) v+m v^{2}}} d v \\
& =\frac{1}{\sqrt{m}}\left[\log \left|2 m v+\left(2 \xi_{\lambda} / \lambda\right)+2 \sqrt{m\left\{m v^{2}+\left(2 \xi_{\lambda} / \lambda\right) v\right\}}\right|\right]_{0}^{\epsilon} \\
& =\frac{1}{\sqrt{(p-1)(1+o(1))}}\left(C_{1, \epsilon}-\log \left(2 \xi_{\lambda} / \lambda\right)\right) .
\end{aligned}
$$

By this and (4.4), we easily obtain $\log \left(\xi_{\lambda} / \lambda\right)=-\sqrt{(p-1) \lambda}(1+o(1)) / 2$ for $\lambda \gg 1$. This implies (2.1). Thus, the proof is complete.

\section{REFERENCES}

[1] H. Berestycki, Le nombre de solutions de certains problèmes semi-linéaires elliptiques, $J$. Funct. Anal. 40 (1981) 1-29. MR607588 (82k:35033)

[2] R. Chiappinelli, Remarks on bifurcation for elliptic operators with odd nonlinearity, Israel J. Math. 65 (1989) 285-292. MR.1005012 (90f:35023)

[3] R. Chiappinelli, On spectral asymptotics and bifurcation for elliptic operators with odd superlinear term, Nonlinear Anal. TMA 13 (1989) 871-878. MR999337(90e:35124)

[4] M. Crandall and P. Rabinowitz, Bifurcation from simple eigenvalues, J. Funct. Anal. 8 (1971) 321-340. MR0288640(44:5836)

[5] J. M. Fraile, J. López-Gómez and J. C. Sabina de Lis, On the global structure of the set of positive solutions of some semilinear elliptic boundary value problems, J. Differential Equations 123 (1995) 180-212. MR1359917 (96j:35073)

[6] M. Holzmann and H. Kielhöfer, Uniqueness of global positive solution branches of nonlinear elliptic problems, Math. Ann. 300 (1994) 221-241. MR1299061 (95m:35068)

[7] P. Rabinowitz, Some global results for nonlinear eigenvalue problems, J. Funct. Anal. 7 (1971) 487-513. MR0301587 (46:745)

[8] T. Shibata, Precise spectral asymptotics for nonlinear Sturm-Liouville problems, J. Differential Equations 180 (2002) 374-394. MR.1894177 (2002m:34124)

[9] T. Shibata, $L^{q}$ spectral asymptotics for nonlinear Sturm-Liouville problems, Differential and Integral Equations 19 (2006) 773-783. MR2235894

Department of Applied Mathematics, Graduate School of Engineering, Hiroshima University, Higashi-Hiroshima, 739-8527, JaPAN 